\title{
SIMULATION AND DESIGN OF EXTRACTION AND SEPARATION FLUIDIC DEVICES
}

\author{
Bijan Mohammadi ${ }^{1}$ And Juan G. Santiago ${ }^{2}$
}

\begin{abstract}
We present the combination of a state control and shape design approaches for the optimization of micro-fluidic channels used for sample extraction and separation of chemical species existing in a buffer solution. The aim is to improve the extraction and identification capacities of electroosmotic micro-fluidic devices by avoiding dispersion of the extracted advected band.

Résumé. Nous présentons la combinaison de deux problèmes d'optimisation et de contrôle d'état pour les configurations de canaux micro-fluidique. Ces dispositifs sont utilisés pour l'extraction et la séparation de composants chimiques présents dans une solution. Le but est, dans un premier temps, l'amélioration de la phase de l'extraction par le contrôle de la dispersion du front. Ceci se fait par l'application d'un champs électrique. L'on s'intèresse ensuite à l'optimisation du canal, permettant la séparation, par une minimisation de la dispersion induite par les coudes.
\end{abstract}

Mathematics Subject Classification. 37NXX, 37N35, 68W25, 74M25, 74PXX, 74SXX.

Received: December 12, 2000. Revised: February 9, 2001.

\section{INTRODUCTION}

Microfluidic channel systems used in bio-analytical applications are fabricated using technologies derived from microelectronics industry including lithography, wet etching and bonding of substrates. Industrial applications of these techniques are quite large and range from DNA sequencing or new drug molecules trials to the detection and identification of pollution in health or water and food quality analysis.

Our aim through this paper is to show two aspects of this problem:

- how to extract a suitable amount of the fluid we want to analyze with minimal band dispersion?

- how to use capillary electrophoresis to perform the separation of the different species present in this band on a compact geometry?

We will see that the first problem leads to an open loop state control problem using the potential difference applied to create mobility in the channel. We will consider a 90 degrees cross configuration representing the beginning of the separation device.

Once the extraction performed, the separation of chemical species, suspended in a liquid buffer, is performed using their electrophoretic mobility. The electric field in these systems is applied in the axis of the channel using electrode immersed at reservoirs at the end of the micro-channels. The ability to discriminate between sample species of nearly equal mobility is enhanced by increasing the channel length [1,2]. Separation capacity

\footnotetext{
Keywords and phrases. Fluidic channels, electroosmosis, optimization and control of distributed systems.

${ }^{1}$ Université Montpellier II, Département de Mathématiques, CC51, 34095 Montpellier Cedex 5, France. e-mail: Bijan.Mohammadi@inria.fr

2 Mechanical Engineering Dept., Stanford University, Stanford 94305-4021, CA, USA. e-mail: Santiago@vk.stanford.edu
} 
increases with the length of the channel $[1,2]$. In order to achieve channel lengths of order $1 \mathrm{~m}$ and yet confine the micro-channel system to a compact configuration with dimensions less than about $10 \mathrm{~cm}$, curved channel geometries are required. Unfortunately, curved channel geometries introduce skews which imply a dispersion of the electrophoretic sample bands in the flow. This curved-channel dispersion has been identified as an important factor in the decrease of separation efficiency of electrophoretic micro-channel systems.

Hence, once the band is extracted with minimal dispersion, we optimize the turns in the separation device in such a way to keep the initial dispersion as much as possible unchanged. The second point in the paper is therefore to show how to use our optimization platform, first designed for aeronautical applications $[5,7]$ for the design of minimal dispersion electrokinetic channels. We noticed that reducing the skew often introduce a new type of residual dispersion associated with band advection away from the channel boundaries. We also noticed that to avoid this effect it is necessary for the channel walls to be as smooth as possible with minimal curvature variation $[3,4]$. We therefore add this constraint to our optimization.

The optimization formulation for such devices has to include the following points:

- minimize the skew due to turns;

- minimize the residual dispersion associated with band advection;

- avoid too much variations in walls curvature;

- maximize the length of the channel;

- minimize the occupied surface.

We show the application of this technique to the design of a 90 and 180 degrees corner minimizing the dispersion of chemical species in motion in the electric field. These turns are important as their combination permits to maximize the length to surface ratio for a channel. Typical cross-section sizes for these channels are $100 \mu \mathrm{m}$ in cross-section width and $10 \mu \mathrm{m}$ in depth.

\section{Governing EQUATIONS}

This problem is multi-model in the sense that several PDEs are involved in the definition of the state variables and the cost function. We will see that different levels of approximation can be introduced for these state equations.

\subsection{Electric field}

The aim is to use the difference in the mobility of the species in an electric field $E(t, x)$. $E$ can be both stationary and unsteady. In this work, we consider only steady electric fields. $E=-\nabla v$ is defined solving a Poisson equation for the potential $v$ :

$$
\begin{aligned}
& \nabla \cdot E=-\Delta v=0, \quad \text { in } \Omega \\
& v\left(\Gamma_{\text {in }}\right)=v_{1}, \quad v\left(\Gamma_{\text {out }}\right)=v_{2}, \\
& v=v_{3} \quad \text { or } \quad \frac{\partial v}{\partial n}=0 \quad \text { on other boundaries. }
\end{aligned}
$$

\subsection{Flow motion}

For typical electrokinetic microchannel applications the observed flow motion has a velocity of about $10^{-4} \mathrm{~m} \mathrm{~s}^{-1}-10^{-3} \mathrm{~m} \mathrm{~s}^{-1}$, channel thickness of $100 \mu \mathrm{m}$ and kinematic viscosity about $10^{-5} \mathrm{~m}^{2} \mathrm{~s}^{-1}$. This leads to Reynolds numbers ranging from 0.001 to 0.01 . Due to spontaneous charge separation that occurs at the channel walls, there is formation of an electric double layer [9]. The typical size of this layer is a few nanometers. The stiffness of this electric double layer makes it hardly computable using classical numerical approaches applied to the Stokes model with a Lorentz force term. However, it is known that at the edge of the double layer the 
flow is parallel and directly proportional to the electric field. The first model describing the flow motion can be therefore the Stokes system with the previous condition as wall-function.

$$
\begin{aligned}
& \frac{\partial U}{\partial t}-\mu \Delta U+\nabla p=0, \quad \text { in the channel } \\
& U=\mu_{e k} E, \quad \text { on the inner and outer walls, } \\
& -\mu \frac{\partial U}{\partial n}+p \cdot n=0, \quad \text { on in and outflow boundaries, }
\end{aligned}
$$

where $\mu_{e k}$ is the electrokinetic mobility of the flow, $\mu$ the dynamical viscosity and $n$ the unit external normal to the boundaries.

\subsection{Reduced models for the flow}

Without pressure gradient, the previous model reduces to two elliptic equations for the velocity components and states that the velocity vector is locally parallel to the walls and proportional to the local electric field $U=\mu_{e k} E \tilde{U}$ with $\tilde{U}$ obtained solving for $\tilde{U}=\left(\tilde{u}_{1}, \tilde{u}_{2}\right)$ :

$$
\begin{gathered}
-\Delta \tilde{u}_{1}=0, \quad-\Delta \tilde{u}_{2}=0, \quad \text { in } \Omega \\
\tilde{U}=\left(\tilde{u}_{1}, \tilde{u}_{2}\right)=\tau=\left(\tau_{1}, \tau_{2}\right), \quad \text { on channel walls, } \quad \frac{\partial \tilde{U}}{\partial n}=0 \quad \text { on other boundaries, }
\end{gathered}
$$

where $\tau$ is the local unit tangent to channel walls. Noticing that the electric field itself is parallel to walls, this means that the velocity is everywhere parallel and proportional to the electric field:

$$
U=\mu_{e k} E
$$

We use this former model in our optimization problem. In addition, this model is interesting for incomplete sensitivity evaluation (see below), where different models are used for the state and gradient computations. In other words, even when using more complex models, we should consider this model as the state equation to be taken into account for sensitivity evaluation.

\subsection{Advection of species}

The motion of a species $a$ at infinite Peclet number by the velocity field $U$ computed above is described by:

$$
\begin{aligned}
a_{t}(x, t)+U(x) \nabla a & =0, \quad \text { in } \Omega, \\
a\left(\Gamma_{\text {inlet }}\right) & =\text { given. }
\end{aligned}
$$

For the shape optimization problem, as we consider the velocity field defined by the stationary electric field, this step is therefore only a post-processing step and is devoted to the qualification of the skew.

\section{Pseudo-time dependent minimization PRoblem}

We consider the following pseudo-time dependent minimization formulation which represents both the state control and shape optimization problems we consider here.

$$
\left\{\begin{array}{l}
\min _{\alpha(t)} J(\alpha(t), q, W), \\
E(\alpha(t), q, W(q, \alpha))=0, \\
g(\alpha(t), q, W) \leq 0 .
\end{array}\right.
$$


where $\alpha(t)$ denotes the control parameter which is here either the potential differences applied during the pinching and pull-back steps, or the turn geometries devoted to keep the dispersion minimal by reducing the skew of the advected band (see below).

In both case, the cost function is given by:

$$
J(\alpha)=\int_{\omega}(\nabla a(\alpha, t) \times U(\alpha))^{2} \mathrm{~d} x,
$$

where $\omega$ denotes the region over which the band extends either after extraction or after the turns. We will see other formulation of this cost function more suitable for sensitivity evaluation in the context of shape design and use of incomplete sensitivities (see below) [7].

Constraints concern admissible amounts of the pinching and pull-back applied potential, the maximum deformation allowed in the turns and also the minimum regularity required for the geometry. $W$ denotes the general state variables vector and $E(\alpha, q, W)$ the state equations described in the previous section. $q$ denotes geometrical entities which depend on the control parameters only in the shape optimization problem.

\subsection{Minimization algorithm}

Problem (6) is closed by considering the following pseudo-time dependent equation for the parameterization $\alpha(t)$ :

$$
\dot{\alpha}+\epsilon \ddot{\alpha}=-G\left(\left(\nabla_{\alpha \alpha} J\right)^{-1}, \nabla_{\alpha} J\right),
$$

where $G$ is a function of the exact or incomplete gradient [7] and of the inverse of the Hessian of the cost function. It also takes into account the projection over the admissible space. This formulation includes most major minimization algorithms [7] such as the steepest descent or the Newton and quasi-Newton methods, or subspace minimization algorithms such as the conjugate gradient method or some global search methods based on dynamic systems such as the heavy ball method [10].

Through this representation, minimization concepts are easy to integrate to a multi-model platform as they consist in adding a new state equation for the parameterization, coupled with the previous existing equations.

The parameterization $\alpha^{0}$ being defined, the dynamical algorithm we use is as follows:

\section{Control and optimization iterations}

1. Compute the gradient: $\frac{\mathrm{d} J^{p}}{\mathrm{~d} \alpha}$, if $\left(\left\|\frac{\mathrm{d} J^{p}}{\mathrm{~d} \alpha}\right\|<T O L\right)$ stop.

2. Define the new admissible parameter variation using (8).

4a. For the shape design problem: smooth shape deformations and deform the mesh (see below).

4b. For the control problem:

Modify the boundary condition for the electric potential field.

5. Compute the new state.

6. Compute the new cost $J^{p+1}$. if $\left(J^{p+1}<T O L\right)$ stop.

6. $p \leftarrow p+1$ and goto 1 .

\section{End of loop.}




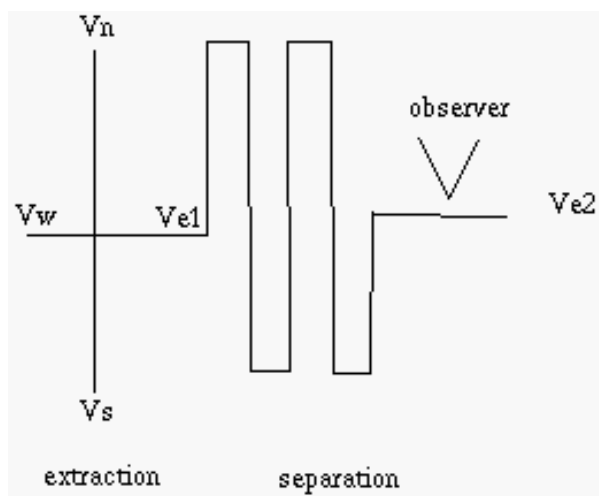

FIgURE 1. Typical geometry for the coupled extraction and separation devices. $V_{n}, V_{s}, V_{e 1}, V_{e 2}$ and $V_{w}$ denote the different potentials applied.
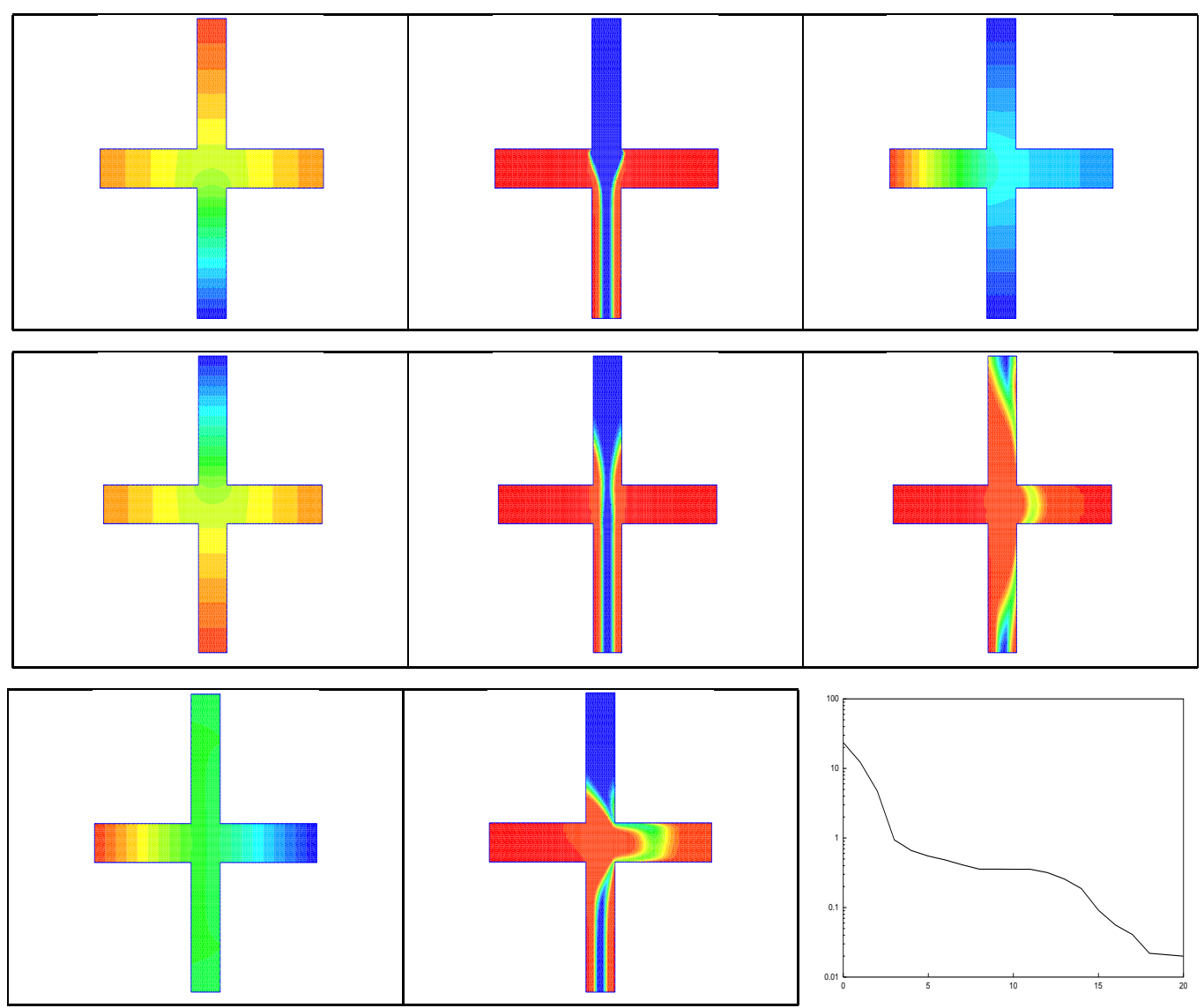

FIGURE 2. Extraction algorithm. Left pictures: electric potential for steps 1-3 above with the control off (no N-S pull-back). Middle pictures: corresponding iso-contour for the advected specie. Right pictures: Last step with the control on, effect on the potential and the extracted band. Lower-right picture: reduction of the band-dispersion by the pull-back potential (more than three orders of magnitude). 


\section{Extraction ALgOrithm}

The first step in our extraction-separation problem, is to extract some amount of the sample we would like to analyze from a continuous flow solution using a secondary flow of buffer solution. This approach have various applications in automatic and predefined analysis of solutions.

A correct simulation of this step requires the following ingredients which aim to recreate the corresponding experimental situations (bellow N-S-E-W denote north-south-east and west):

- 1a. Evaluation of the N-S electric potential plus a E-W pinching solving:

$$
\begin{gathered}
-\Delta v=0, \quad \text { in extraction domain } \\
v\left(\Gamma_{\text {north }}\right)=V_{n}, \quad v\left(\Gamma_{\text {south }}\right)=V_{s}, \\
v\left(\Gamma_{\text {east }}\right)=v\left(\Gamma_{\text {west }}\right)=V_{e}, \quad \text { and } \quad \frac{\partial v}{\partial n}=0 \quad \text { on other boundaries, }
\end{gathered}
$$

here we take $V_{n} / V_{s}=2$ and $V_{e} / V_{s}=V_{w} / V_{s}=1.9$.

- 1b. N-S advection of the species by the main solution using the electric field of 1a, during a time enough long for the flow to be steady.

- 2a. Evaluation of the N-S pull-back electric potential with again a E-W pinching by solving (9) where we take $V_{n} / V_{s}=0.5$ and $V_{e} / V_{s}=V_{w} / V_{s}=0.95$.

- 2b. N-S pull-back advection using the electric field of 2a for a time enough long for the flow to move back at least the channel thickness.

- 3a. Evaluation of the W-E electric potential plus N-S pull-back (the control) by solving (9) where we take $V_{n} / V_{w}=V_{s} / V_{w}=C$ and $V_{e} / V_{w}=0.5$.

The optimal pull-back for this step has been found to be given by $C=0.4$.

- 3b. W-E advection using the electric field of $3 a$.

- 4. Evaluation of the amount of band dispersion after the cross to qualify the separation quality using (7).

In this algorithm, the first four steps are devoted to simulate the situation of a convecting flow, from which it is suitable to extract a specie band with minimal dispersion. The tools to minimize the dispersion are pinching and pull-back potentials. Here, after setting up the pinching and pull-back for step 1 and 2 on some predefined values, we tried to find the final optimal pull-back potential for step 3.

\subsection{Sensitivity analysis by the complex variable method}

As, in the control problem, the number of control parameter is small, as seen above, finite differences can be used to find the sensitivity of the cost function with respect to control parameters variations. Another alternative, which does avoid some limitations of finite differences is the complex variable method [11]. A Taylor expansion in the complex plane enables for the definition of the gradient:

$$
J(\alpha+i \varepsilon)=J(\alpha)+i \varepsilon \frac{\mathrm{d} J}{\mathrm{~d} \alpha}-\frac{\varepsilon^{2}}{2} \frac{\mathrm{d}^{2} J}{\mathrm{~d} \alpha^{2}}+\varepsilon^{2} o(1),
$$

therefore

$$
\frac{\mathrm{d} J}{\mathrm{~d} \alpha}=\frac{\operatorname{Im}(J(\alpha+i \varepsilon))}{\varepsilon}+\varepsilon^{2} o(1) .
$$

We can see that there is no substraction in the evaluation and therefore the choice of $\varepsilon$ not as critical as in finite differences:

$$
\frac{\mathrm{d} J}{\mathrm{~d} \alpha}=\frac{J(\alpha+\varepsilon)-J(\alpha)}{\varepsilon} .
$$


In practice, this methods only requires a redefinition of all real variables of the computer programs in the control loop as complex. The complexity of the approach is comparable to first order (forward) finite differences despite the fact that complex operations and storages require twice effort than for reals but the evaluation of the functional after a small change in the complex plan will not greatly affect the real part:

$$
\operatorname{Re}(J(x+i \varepsilon)) \sim J(x)
$$

therefore only one evaluation is necessary to get $J$ and $\mathrm{d} J / \mathrm{d} \alpha$.

\section{Shape DESign FOR SEPARATION}

The shape optimization problem can be formulated as in 6 where $\alpha=x \in \mathcal{O} \subset \mathbb{R}^{n_{c}}$ denotes the our CADFree [5] parameterization of the shape, $\mathcal{O}$ being the admissible space, $n_{c}$ the size of the design space and we denote by $q(x)$ all geometrical entities (normals, surfaces,...) which depend this time on the control parameters.

\section{CAD-FreE SHAPE AND MESH DEFORMATION TOOLS}

The shape deformation is done in a CAD-Free framework [7] in the sense that the only entity known during optimization is the mesh. This parameterization has several characteristics:

1. All the nodes on the inner wall of the channel are control points. More precisely, we use the local normal to the inner channel wall and specify the deformations in the direction of this normal $n(x)$. Hence, for a curve $\gamma(x)$, a deformation of amount $f(x)$, defined for each nodes, leading to the deformed curve $\tilde{\gamma}(x)$, can be expressed in the normal direction to $\gamma$ by:

$$
\tilde{\gamma}(x)=\gamma(x)+f(x) n(\gamma(x)) .
$$

2. To avoid oscillations, a "local" smoothing operator is defined over the shape.

The reason for this is that the gradient has necessarily less regularity than the parameterization. Indeed, suppose that the cost function is a quadratic function of the parameterization: $J(x)=\|A x-b\|^{2}$ with $x \in$ $H^{1}(\Gamma), A \in H^{-1}(\Gamma)$ and $b \in L^{2}(\Gamma)$. The gradient $\mathrm{d} J / \mathrm{d} x=(2(A x-b) A) \in V$ with $H^{-1}(\Gamma) \subset V \subset L^{2}(\Gamma)$. Hence, any parameterization variation using $\mathrm{d} J / \mathrm{d} x$ as descent direction will have less regularity than $x$ : $\delta x=-\rho \mathrm{d} J / \mathrm{d} x=-\rho(2(A x-b) A) \in V$, where $H^{-1}(\Omega) \subset V \subset L^{2}(\Omega)$. We need therefore to project into $H^{1}(\Omega)$ using the localized solution of a second order elliptic system in regions where the deformation is found to be not enough smooth.

$$
\begin{gathered}
(I-\varepsilon \Delta) \delta \tilde{x}=\delta x, \\
\delta \tilde{x}=\delta x=0 \quad \text { where constrained, }
\end{gathered}
$$

where $\delta \tilde{x}$ is the smoothed shape variation for the shape nodes and $\delta x$ is the variation given by the optimization tool. By "local" we mean that if the predicted shape is locally smooth, it remains unchanged during this step and $\varepsilon$ is set to zero for these regions if,

$$
\frac{\delta_{i j}(\delta x)}{(\delta x)_{T}}<T O L
$$

where $\delta_{i j}(\delta x)$ is the difference between the variations of the two nodes of each boundary edge and $(\delta x)_{T}$ the mean variation on this edge and $T O L$ is a regularity tolerance factor.

Once the shape deformation defined, it is propagated over the computational domain using elasticity based procedure as described in [7]. 


\section{Sensitivity And incomplete Sensitivities}

Consider the general simulation loop, involved in (6), leading from shape parameterization to the cost function:

$$
J(x): x \rightarrow q(x) \rightarrow W(q(x)) \rightarrow J(x, q(x), W(q(x)))
$$

The Jacobian of $J$ is given by:

$$
\frac{d J}{d x}=\frac{\partial J}{\partial x}+\frac{\partial J}{\partial q} \frac{\partial q}{\partial x}+\frac{\partial J}{\partial W} \frac{\partial W}{\partial q} \frac{\partial q}{\partial x}
$$

In most applications, the cost function is, or can be reformulated, to have the following characteristics:

- The cost function $J$ and the parameterization $x$ are defined on the shape (or a same part of it),

- $J$ is of the form

$$
J(x)=\int_{\text {shape or part of the shape }} f(x, q) g(W) \mathrm{d} \gamma,
$$

which means that it involves a product of geometrical and state based functions,

We have shown that for such cost functions, the sensitivity with respect to the state can be neglected in regions where the curvature of the shape is not too large [5-8]. This means that only the shape deformation tool has to be differentiated and not the whole simulation loop, in particular neither the mesh deformation or the state equation solver have to be linearized [6]. Hence, we consider the following approximation for the gradient:

$$
\frac{\mathrm{d} J}{\mathrm{~d} x} \sim \frac{\partial J}{\partial x}+\frac{\partial J}{\partial q} \frac{\partial q}{\partial x}
$$

The argument above means that cost function (7) based on domain integral is not suitable for cheap sensitivity evaluation. The first approximation is to consider the cost function only in regions of space where the channel has no turn and therefore where $U$ is constant:

$$
J(x)=\int_{\Omega}\left(\frac{\partial a(x, T)}{\partial n}\right)^{2} \mathrm{~d} x
$$

where $n$ is the normal direction to the local channel walls.

Another formulation for the cost function is to ask for all particles traveling on characteristics to have the same migration time. Hence, the cost function is given by:

$$
J(x)=\left(\int_{\chi} \frac{\mathrm{d} s}{U}-\int_{\chi^{\prime}} \frac{\mathrm{d} s}{U}\right)^{2}
$$

for any couple of characteristics $\chi$ and $\chi^{\prime}$ linking the outlet to the inlet. But again, the cost function is over the whole space. However, we can consider only a few characteristics. The two main characteristics are those defined by the internal and external walls of the channel:

$$
J(x)=\left(\int_{\Gamma_{i}} \frac{\mathrm{d} s}{U \cdot \tau}-\int_{\Gamma_{o}} \frac{\mathrm{d} s}{U \cdot \tau}\right)^{2}
$$

where $\Gamma_{i}$ is the inner wall and $\Gamma_{o}$ the outer wall of the channel. This last formulation is interesting as it only involves boundaries which we know to be suitable for the application of our incomplete sensitivity. 

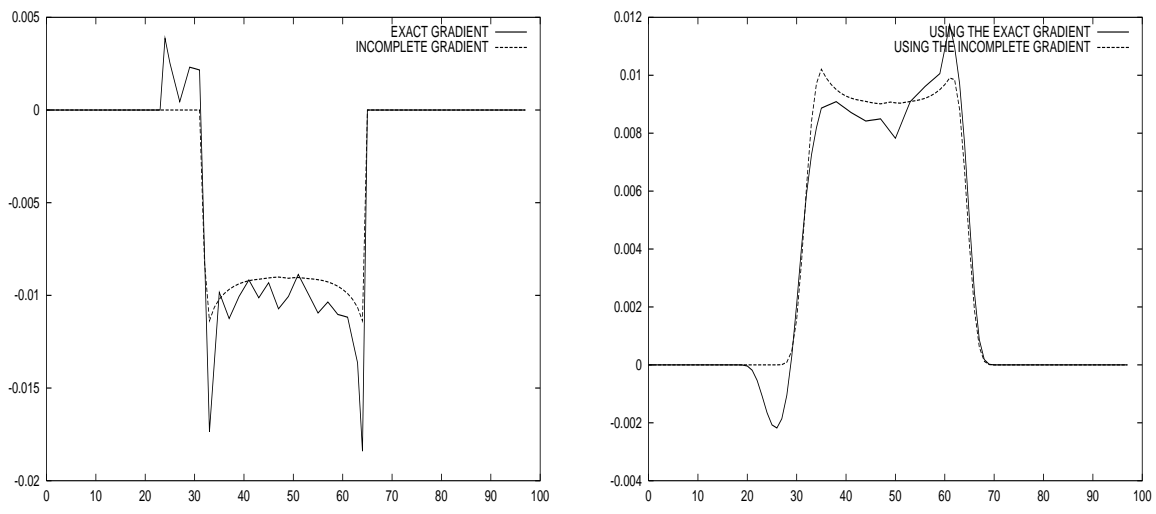

Figure 3. Sensitivity evaluation around the initial 90 degree turn for control points along the inner channel wall. Comparison between the exact and incomplete gradient evaluations (left). The deformations obtained using these gradients (right).

For shape optimization, we notice that the residual band advection dispersion away from walls increases with the variation of the wall curvature. We therefore add the following constraint to the cost function (14) above:

$$
J(x) \leftarrow J(x)+\left(\int_{\Gamma_{i}}\left\|\frac{\partial n}{\partial s}\right\|-\int_{\Gamma_{i}^{0}}\left\|\frac{\partial n}{\partial s}\right\|\right)^{2}+\left(\int_{\Gamma_{o}}\left\|\frac{\partial n}{\partial s}\right\|-\int_{\Gamma_{o}^{0}}\left\|\frac{\partial n}{\partial s}\right\|\right)^{2},
$$

where 0 denotes initial inner and outer walls.

Two other types of geometrical constraints concern the amplitude of the deformations and the regularity of the deformed shape. In the first constraint, shape variations are allowed between two limiting curves.

We said that cost function (14) is suitable for the application of incomplete sensitivities. We can increase direct geometrical contributions by the fact that the velocity is parallel to the walls. The cost function we consider for derivation is therefore:

$$
J(x)=\left(\int_{\Gamma_{i}} \frac{\mathrm{d} s}{\vec{\tau} \mu_{e k}|E|}-\int_{\Gamma_{o}} \frac{\mathrm{d} s}{\vec{\tau} \mu_{e k}|E|}\right)^{2},
$$

where $\vec{\tau}$ is the local unit tangent vector to the wall.

To evaluate the accuracy of these gradients, we compare the results obtained with this approximation of the gradients with those coming from finite differences. This incomplete sensitivity evaluation possibility shows the importance of redefining cost functions as boundary integrals when possible and to bring as close as possible the cost function and control definition locations. In fact, optimization becomes possible for the configurations for which simulation is affordable as the cost of simulation and design becomes equivalent.

\section{Conclusion}

The combination of state control and shape optimization techniques for minimal dispersion extraction and separation fluidic devices has been presented. Incomplete sensitivity approach has been used after a redefinition of the cost function for the shape design problem. The same pseudo-unsteady optimization algorithm has been used for both the control and design problems. Current effort concerns the extension of the state equation to the solution of variable conductivity samples introducing local pressure gradients and discontinuous solutions in the field. 

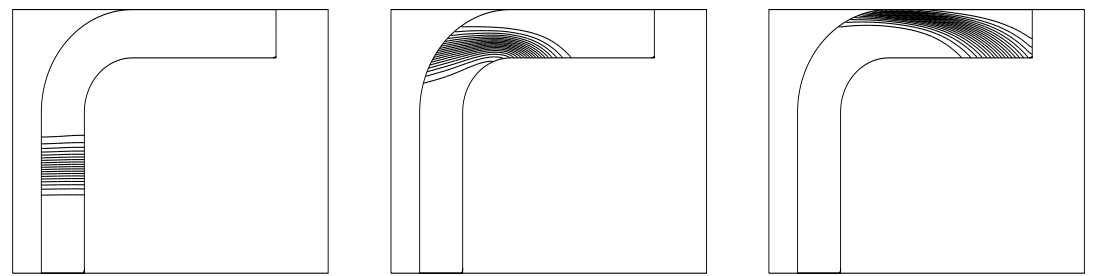

FiguRE 4. Initial shape for the 90 degrees turn: effect of the turn on the advected species.
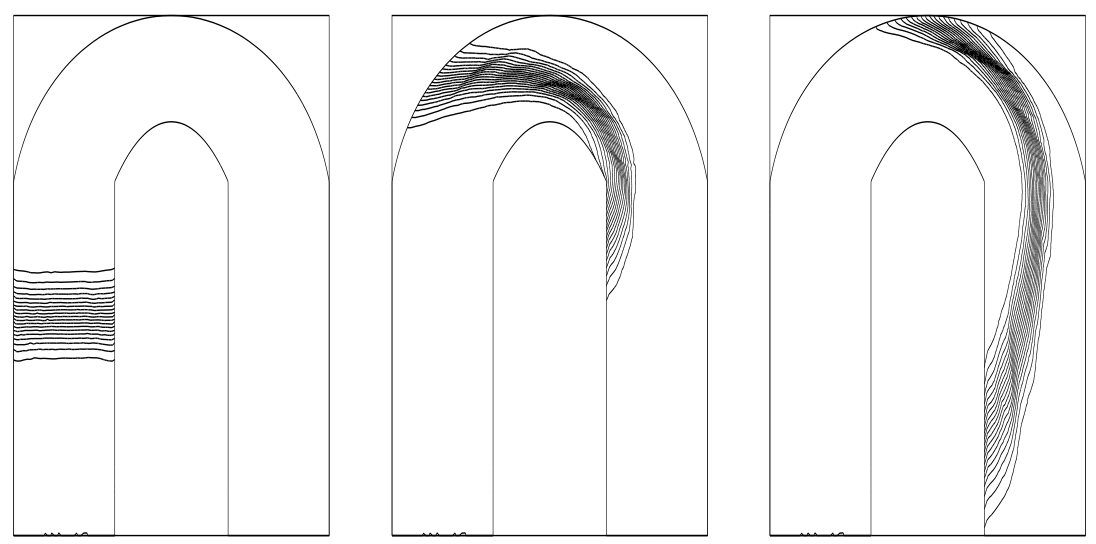

Figure 5. Initial shape for the 180 degrees turn: effect of the turn on the advected species.
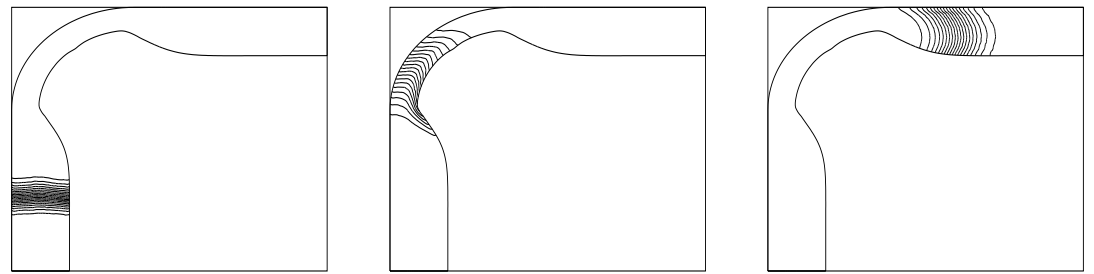

FiguRE 6. Optimized shape for the 90 degree turn with both the inner and outer walls moving.
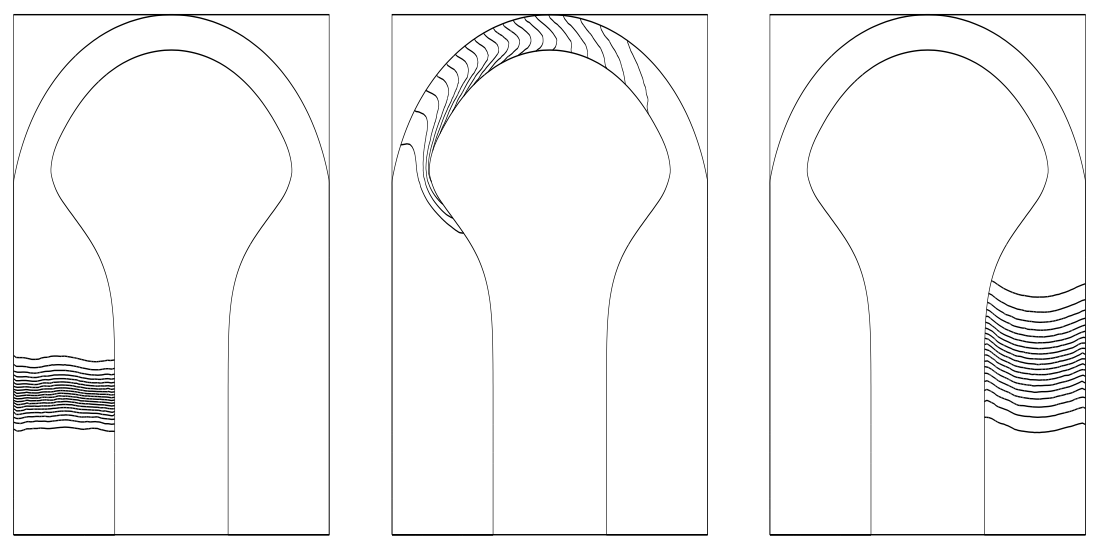

FiguRE 7. Optimized shape for the 180 degree turn with both the inner and outer walls moving. 
Acknowledgements. This work has been supported by INRIA, the Center for Turbulence Research at Stanford university and DARPA. Many thanks to Profs. P. Moin, B. Perthame and O. Pironneau for their interests in this work realization. Many thanks to M. Bautista, D. Spinks and M. Fatica for their assistance.

\section{REFERENCES}

[1] C.T. Culbeston, S.C. Jacobson and J. Ramsey, Dispersion sources for compact geometries on microchips. Analytical Chemistry 70 (1998) 3781-3789.

[2] J.I. Molho, A.E. Herr, B.P. Mosier, J.G. Santiago, T.W. Kenny, R.A. Brennen and G.B. Gordon, Designing corner compensation for electrophoresis in compact geometries, in Proc. Micro total analysis systems, Enschelde, The Netherlands, May 14-18, 2000. Kluwer Academic, Hingham (2000) 287-290.

[3] B. Mohammadi, J.I. Molho and J.G. Santiago, Incomplete sensitivities in the design of minimal dispersion fluidic channels. Comp. Meth. Appl. Mech. Eng. (submitted).

[4] B. Mohammadi, J.I. Molho and J.G. Santiago, Optimization of turn geometries for on-chip electrophoresis. Analytical Chemestry 73 (2001) 1350-1360.

[5] B. Mohammadi, Practical applications to fluid flows of automatic differentiation for design problems. VKI Lecture Series 4S-05 (1997) 55-84.

[6] B. Mohammadi, A new optimal shape design procedure for inviscid and viscous turbulent flows. Internat. J. Numer. Methods Fluids 25 (1997) 183-203.

[7] B. Mohammadi and O. Pironneau, Applied Shape Design for Fluids. Oxford Univ. Press, Oxford (2001).

[8] B. Mohammadi, Flow control and shape optimization in aeroelastic configurations. AIAA 99-0182 (1999).

[9] R.F. Probstein, Physicochemical hydrodynamics. Wiley, New York (1995).

[10] H. Attouch and R. Cominetti, A dynamical approach to convex minimization coupling approximation with the steepest descent method. J. Differential Equations 128 (1996) 519-540.

[11] W. Squire and G. Trapp, Using complex variables to estimate derivatives of real functions. SIAM Review 10 (1998) 110-112.

To access this journal online: www.edpsciences.org 